\title{
Correction to: International Symposium on Mathematics, Quantum Theory, and Cryptography
}

Tsuyoshi Takagi, Masato Wakayama, Keisuke Tanaka,

Noboru Kunihiro, Kazufumi Kimoto, and Yasuhiko Ikematsu

\section{Correction to:}

T. Takagi et al. (eds.), International Symposium

on Mathematics, Quantum Theory, and Cryptography,

Mathematics for Industry 33,

https://doi.org/10.1007/978-981-15-5191-8

The original version of the book was published with incorrect part names in the website for second part FM now updated with correct title. This has been corrected in the updated version. 